\title{
Target detection and localization method for distributed monopulse arrays in the presence of mainlobe jamming
}

\author{
Qing Sun ${ }^{1}$, Qiliang Zhang ${ }^{1 *}$ (D), Xueyu Huang ${ }^{1}$ and Qian Gao²
}

\begin{abstract}
In this paper, we propose a target detection and localization method on distributed monopulse arrays for tracking radar. An optimized mainlobe jamming (MLJ) cancellation filter was designed by maximizing the power ratio of the received siackgnal to the jamming-plus-noise. By exploiting the different correlation characteristics between the target echo and MLJ on distributed antennas, the designed filter is able to cancel MLJ and maintain the target echo. By applying the identical filter on sum-difference beams, MLJ can be cancelled, and the monopulse ratio can be maintained simultaneously. Hence, we simply detect and locate the target on the filtering output of sum-difference beams according to the monopulse principle. Monte Carlo simulations demonstrated that the proposed filter outperforms the conventional algorithms.
\end{abstract}

Keywords: Mainlobe jamming, Distributed monopulse antennas, Sum-difference network, Spatial filter

\section{Introduction}

The monopulse technique, which can be easily realized on a lens, reflector, or array antenna, is widely utilized in tracking radar systems [1]. Each type of antennas will simultaneously generates sum and difference beams using a cascaded monopulse network. Target detection and localization can be carried out on the output of the sum-difference beams with satisfactory performance in the absence of external jamming [1]. However, the performance degrades greatly when sidelobe jamming (SLJ) or mainlobe jamming (MLJ) is received by the beams due to the decreased signal to jamming-plus-noise ratio (SJNR) $[2,3]$. In the case of SLJ, this problem can be solved using the sidelobe cancellation (SLC) technique $[4,5]$ with the aid of auxiliary antennas. In contrast, the SLC technique will not work in the case of MLJ since the gain of auxiliary antennas in MLJ is much smaller than that of the mainlobe.

To address MLJ, the mainlobe cancellation (MLC) technique for two-dimensional monopulse antennas has been studied in [6-8]. This technique filters out MLJ

*Correspondence: zql3884@163.com

${ }^{1}$ Air Force Engineering University, 710051 Xi'an, China

Full list of author information is available at the end of the article on monopulse beams, including sum $(\Sigma)$, delta-elevation $\left(\Delta_{e}\right)$, delta-azimuth $\left(\Delta_{a}\right)$, and delta-delta $\left(\Delta_{\Delta}\right)$. The monopulse ratio is maintained when MLC is implemented. Hence, the target angle can be accurately estimated from the outputs of the filter according to the monopulse principle. However, this technique can only work under the condition that there is just one MLJ since each MLJ filter has only two input channels. Its performance would degrade greatly when multiple MLJs arrive from different directions. To cope with multiple MLJs, an array antenna and adaptive beamforming algorithm [9] should be employed instead of the adaptive monopulse network. When the adaptive array performs MLC, the shape of the mainlobe is distorted greatly and the gain of the target echo becomes very small [10]. Another MLC technique is proposed in $[11,12]$. It employs a largescale auxiliary array for the main antenna and constructs an adaptive filter using the criterion of minimizing the mean square error (MMSE) or maximizing SJNR (MSJNR) for the combination of the main antenna and the auxiliary array. The distribution of the antenna elements on the auxiliary array is optimized using a genetic algorithm. This technique generates rather narrow notch in the MLJ direction by adapting the large-scale auxiliary 
array. Hence, the target in another direction in the mainlobe can be separated and detected. Nevertheless, the technique is not suitable for deploying a super large-scale auxiliary array for tracking radar in practical scenarios.

Different from these filtering techniques, a novel target detection method has been proposed based on the fact that the correlation coefficients of the target echo and MLJ are quite different from each other on distributed antennas [13]. This method can detect the target even in the case when the target echo and MLJ come from the same direction. However, the detection performances degrades greatly when the target echo overlaps with MLJ because the correlation coefficient of the mixture is much larger than that of the isolated target echo. Thus, the overlapped signal may be denied as MLJ with a large probability.

Inspired by [13], we proposed a MLJ filtering method in our previous study [14]. The proposed method can separate the target and MLJ even when they are superposed in both the time and space domains. However, the filter is designed based on the estimation of the MLJ subspace, which is difficult in practical scenarios. To solve this problem, we propose a new MLJ cancellation method without MLJ subspace estimation, which is applicable to many MLJ scenarios. MLJ can be cancelled, and the monopulse ratio can be maintained by simultaneously performing the identical filter on $\Sigma$, $\Delta_{e}$, and $\Delta_{a}$ beams of distributed monopulse antennas (DMA). Thus, the angle coordinate can be estimated from the filtered output according to the monopulse principle.

The rest of this paper is organized as follows. In Section 2, the MLC techniques for a single antenna are introduced, and their performances are analyzed. In Section 3, a MLJ filter is designed, and the corresponding target detection and localization methods are proposed. In Section 4, numerical simulations are presented for typical scenarios. Finally, conclusions are provided in Section 5.

\section{Background}

The monopulse-network cascaded array antenna and completely adaptive array antenna are typical monopulse antenna architectures for modern tracking radars. The adaptive MLC technique for a monopulse network proposed in [6] and the optimal filtering algorithm for an array antenna proposed in [9] are outlined here, and their performances are analyzed theoretically.

\subsection{MLC for a monopulse network}

\subsubsection{One-dimensional monopulse network}

Given an $N$-element uniform linear array (ULA) with half-carrier wavelength spacing illustrated in Fig. 1a, the steering vector in direction $\theta$ can be expressed as

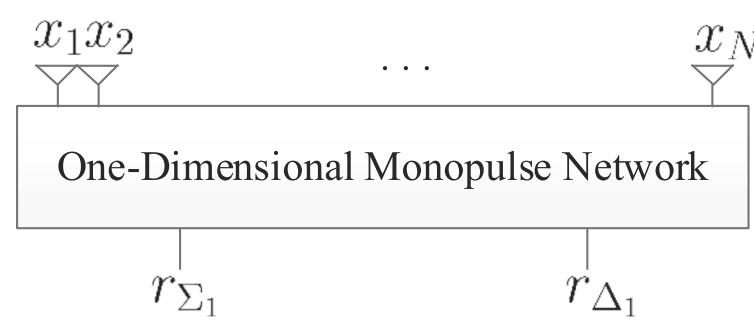

(a)

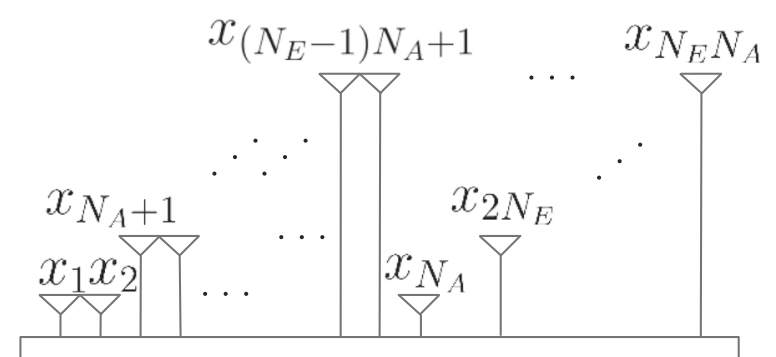

Two-Dimensional Monopulse Network

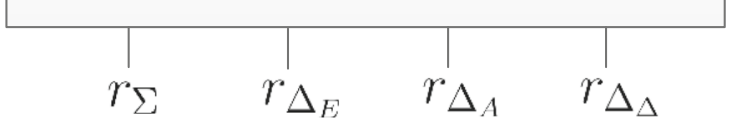

(b)

Fig. 1 Monopulse Network on a Linear Array. (a) One dimensional array, (b) Two dimensional array

$$
\boldsymbol{a}_{\theta, N}=\left[1, e^{\mathrm{j} \pi \sin \theta}, \cdots, e^{\mathrm{j}(N-1) \pi \sin \theta}\right]^{T} .
$$

The monopulse network will simultaneously formulate the sum $\left(\Sigma_{1}\right)$ and difference $\left(\Delta_{1}\right)$ beams in the predicted target direction $\theta_{P}$, whose sidelobes are usually controlled by employing Taylor and Bayliss windows $[15,16]$. Thus, the beam formers of $\Sigma_{1}$ and $\Delta_{1}$ can be written as

$$
\begin{aligned}
& \boldsymbol{w}_{\Sigma_{\theta_{P}, N}}=\boldsymbol{a}_{\theta_{P}, N} \circ \boldsymbol{w}_{T, N}, \\
& \boldsymbol{w}_{\Delta_{\theta_{P}, N}}=\boldsymbol{a}_{\theta_{P}, N} \circ \boldsymbol{w}_{B, N},
\end{aligned}
$$

where $\circ$ denotes the Hadamard product of two vectors, whereas $\boldsymbol{w}_{T, N}$ and $\boldsymbol{w}_{B, N}$ denote the Taylor and Bayliss window vectors with $N$ elements, respectively.

Then, the antenna gains of the $\Sigma_{1}$ and $\Delta_{1}$ beams in direction $\theta$ are

$$
\begin{aligned}
& g_{\Sigma_{\theta_{P}, N}}(\theta)=\boldsymbol{w}_{\Sigma_{\theta_{P}, N}}^{H} \boldsymbol{a}_{\theta, N}=\boldsymbol{w}_{T, N}^{H} \tilde{\boldsymbol{a}}_{\theta_{P}, \theta, N}, \\
& g_{\Delta_{\theta_{P}, N}}(\theta)=\boldsymbol{w}_{\Delta_{\theta_{P}, N}}^{H} \boldsymbol{a}_{\theta, N}=\boldsymbol{w}_{B, N}^{H} \tilde{\boldsymbol{a}}_{\theta_{P}, \theta, N},
\end{aligned}
$$

where

$$
\begin{aligned}
\tilde{\boldsymbol{a}}_{\theta P}, \theta, N & \triangleq \\
& \boldsymbol{a}_{\theta, N}^{*} \circ \boldsymbol{a}_{\theta, N} \\
& =\left[1, e^{\mathrm{j} \pi\left(\sin \theta-\sin \theta_{P}\right)}, \ldots, e^{\mathrm{j}(N-1) \pi\left(\sin \theta-\sin \theta_{P}\right)}\right]^{T},
\end{aligned}
$$


is defined as the relative steering vector (RSV) of the monopulse network.

\subsubsection{Two-dimensional monopulse network}

For the planar array with half-carrier wavelength spacing in each row and column direction illustrated in Fig. 1b, the steering vector corresponding to elevation $\theta_{e}$ and azimuth $\theta_{a}$ can be written as

$$
\boldsymbol{v}_{\theta_{e}, \theta_{a}}=\boldsymbol{a}_{\theta_{e}, N_{e}} \otimes \boldsymbol{a}_{\theta_{a}, N_{a}},
$$

where $\otimes$ denotes the Kronecker product of two vectors, and $N_{e}$ and $N_{a}$ denote the number of rows and columns on the array, respectively.

Thus the signal received by the array is

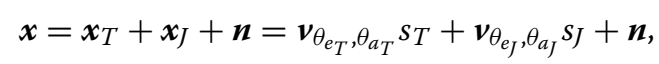

where $s_{T}$ and $s_{J}$ denote the target echo and jamming signal arriving from the directions $\left(\theta_{e_{T}}, \theta_{a_{T}}\right)$ and $\left(\theta_{e_{J}}, \theta_{a_{J}}\right)$ with the power $P_{S}$ and $\sigma_{J}^{2}$ respectively. Moreover, $\boldsymbol{n}$ is an independent and identically distributed (i.i.d.) Gaussian random vector with zero mean and covariance matrix $\boldsymbol{R}_{\boldsymbol{n}}=\sigma^{2} \boldsymbol{I}_{N_{e} N_{a} \times N_{e} N_{a}}$.

For two-dimensional monopulse network, the beam formers of $\Sigma, \Delta_{e}, \Delta_{a}$, and $\Delta_{\Delta}$ beams can be expressed as

$$
\begin{aligned}
\boldsymbol{w}_{\Sigma} & =\boldsymbol{w}_{\Sigma_{\theta_{e_{P}}, N_{e}}} \otimes \boldsymbol{w}_{\Sigma_{\theta_{a_{P}}, N_{a}}}, \\
\boldsymbol{w}_{\Delta_{e}} & =\boldsymbol{w}_{\Delta_{\theta_{e}, N_{e}}} \otimes \boldsymbol{w}_{\Sigma_{\theta_{a_{P}}, N_{a}}}, \\
\boldsymbol{w}_{\Delta_{a}} & =\boldsymbol{w}_{\Sigma_{\theta_{e_{P}}, N_{e}}} \otimes \boldsymbol{w}_{\Delta_{\theta_{a_{P}}, N_{a}}}, \\
\boldsymbol{w}_{\Delta_{\Delta}} & =\boldsymbol{w}_{\Delta_{\theta_{e}, N_{e}}} \otimes \boldsymbol{w}_{\Delta_{\theta_{a_{P}}, N_{a}}},
\end{aligned}
$$

where the monopulse axis points at elevation $\theta_{e_{P}}$ and azimuth $\theta_{a_{P}}$.

Then the outputs of the monopulse beams are

$$
r_{\Sigma}=\boldsymbol{w}_{\Sigma}^{H} \boldsymbol{x}, r_{\Delta_{e}}=\boldsymbol{w}_{\Delta_{e}}^{H} \boldsymbol{x}, r_{\Delta_{a}}=\boldsymbol{w}_{\Delta_{a}}^{H} \boldsymbol{x}, r_{\Delta_{\Delta}}=\boldsymbol{w}_{\Delta_{\Delta}}^{H} \boldsymbol{x} .
$$

The gains in the direction $\left(\theta_{e}, \theta_{a}\right)$ of the monopulse beams formed according to (9)-(12) can be derived from (4), (5) and (7) as

$$
\begin{gathered}
g_{\Sigma}\left(\theta_{e}, \theta_{a}\right)=\boldsymbol{w}_{\Sigma}^{H} \boldsymbol{v}_{\theta_{e}, \theta_{a}}=g_{\Sigma_{\theta_{e}, N_{e}}}\left(\theta_{e}\right) g_{\Sigma_{\theta_{a_{p}}, N_{a}}}\left(\theta_{a}\right), \\
g_{\Delta_{e}}\left(\theta_{e}, \theta_{a}\right)=\boldsymbol{w}_{\Delta_{e}}^{H} \boldsymbol{v}_{\theta_{e}, \theta_{a}}=g_{\Delta_{\theta_{e}, N_{e}}}\left(\theta_{e}\right) g_{\Sigma_{\theta_{a_{p}, N_{a}}}}\left(\theta_{a}\right), \\
g_{\Delta_{a}}\left(\theta_{e}, \theta_{a}\right)=\boldsymbol{w}_{\Delta_{a}}^{H} \boldsymbol{v}_{\theta_{e}, \theta_{a}}=g_{\Sigma_{\theta_{e_{p}}, N_{e}}}\left(\theta_{e}\right) g_{\Delta_{\theta_{a_{p}}, N_{a}}}\left(\theta_{a}\right), \\
g_{\Delta_{\Delta}}\left(\theta_{e}, \theta_{a}\right)=\boldsymbol{w}_{\Delta_{\Delta}}^{H} \boldsymbol{v}_{\theta_{e}, \theta_{a}}=g_{\Delta_{\theta_{e}, N_{e}}}\left(\theta_{e}\right) g_{\Delta_{\theta_{a_{p}}, N_{a}}}\left(\theta_{a}\right) .
\end{gathered}
$$

\subsubsection{Adaptive filtering for monopulse networks}

Adaptive MLC filters for monopulse networks (AMFMNs) have been studied in [6-8]. These filters can be expressed as

$$
\begin{aligned}
& \tilde{r}_{\Sigma_{e}}=r_{\Sigma}-w_{a} r_{\Delta_{a}}, \tilde{r}_{\Delta_{e}}=r_{\Delta_{e}}-w_{a} r_{\Delta_{\Delta}}, \\
& \tilde{r}_{\Sigma_{a}}=r_{\Sigma}-w_{e} r_{\Delta_{e}}, \tilde{r}_{\Delta_{a}}=r_{\Delta_{a}}-w_{e} r_{\Delta_{\Delta}},
\end{aligned}
$$

where $\tilde{r}_{\Sigma_{e}}, \tilde{r}_{\Sigma_{a}}, \tilde{r}_{\Delta_{e}}$, and $\tilde{r}_{\Delta_{a}}$ are the outputs of the filters.

The coefficients of these filters are determined as

$$
\begin{aligned}
w_{a} & =\frac{\frac{E\left[r_{\Sigma} r_{\Delta_{a}}^{*}\right]}{E\left[r_{\Delta_{a}} r_{\Delta_{a}}^{*}\right]}+\frac{E\left[r_{\Delta_{e}} r_{\Delta_{\Delta}}^{*}\right]}{E\left[r_{\Delta_{\Delta}} r_{\Delta_{\Delta}}^{*}\right]}}{2}=\frac{\frac{\boldsymbol{w}_{\Sigma}^{H} \boldsymbol{R} \boldsymbol{w}_{\Delta_{a}}}{\boldsymbol{w}_{\Delta_{a}}^{H} \boldsymbol{R} \boldsymbol{w}_{\Delta_{a}}}+\frac{\boldsymbol{w}_{\Delta e}^{H} \boldsymbol{R} \boldsymbol{w}_{\Delta_{\Delta}}}{\boldsymbol{w}_{\Delta_{\Delta}}^{H} \boldsymbol{R} \boldsymbol{w}_{\Delta_{\Delta}}}}{2} \\
\approx & \frac{g_{\Sigma_{\text {}_{a_{P}, N_{a}}}}\left(\theta_{a_{J}}\right)}{g_{\Delta_{\theta_{P}, N_{a}}}\left(\theta_{a_{J}}\right)},
\end{aligned}
$$

$$
\begin{aligned}
w_{e} & =\frac{\frac{E\left[r_{\Sigma} r_{\Delta_{e}}^{*}\right]}{E\left[r_{\Delta_{e}} r_{\Delta_{e}}^{*}\right]}+\frac{E\left[r_{\Delta_{a}} r_{\Delta_{\Delta}}^{*}\right]}{E\left[r_{\Delta_{\Delta}} r_{\Delta_{\Delta}}^{*}\right]}}{2}=\frac{\frac{\boldsymbol{w}_{\Sigma}^{H} \boldsymbol{R} \boldsymbol{w}_{\Delta_{e}}}{\boldsymbol{w}_{\Delta_{e}}^{H} \boldsymbol{R} \boldsymbol{w}_{\Delta_{e}}}+\frac{\boldsymbol{w}_{\Delta a}^{H} \boldsymbol{R} \boldsymbol{w}_{\Delta_{\Delta}}}{\boldsymbol{w}_{\Delta_{\Delta}}^{H} \boldsymbol{R} \boldsymbol{w}_{\Delta_{\Delta}}}}{2} \\
\approx & \frac{g_{\Sigma_{\theta_{e_{P}}, N_{e}}}\left(\theta_{e_{J}}\right)}{g_{\Delta_{\theta_{P}, N_{e}}}\left(\theta_{e_{J}}\right)}
\end{aligned}
$$

where $\boldsymbol{R}$ is the covariance of jamming-pluse-noise derived from (8) with the form

$$
\begin{aligned}
\boldsymbol{R} & =E\left[\left(\boldsymbol{v}_{\theta_{e j}, \theta_{a J}} s_{J}+\boldsymbol{n}\right)\left(\boldsymbol{v}_{\theta_{e j}, \theta_{a J}} s_{J}+\boldsymbol{n}\right)^{H}\right] \\
& =\boldsymbol{v}_{\theta_{e j}, \theta_{a J}}^{H} \boldsymbol{v}_{\theta_{e j}, \theta_{a J}} \sigma_{J}^{2}+\sigma^{2} \boldsymbol{I}_{N_{e} N_{a} \times N_{e} N_{a} .} .
\end{aligned}
$$

It has been proved in [6] that the filters in (17) can hold the monopulse ratio while performing MLC. Hence, the target direction can be easily estimated from the outputs of the MLC filters according to monopulse principle.

\subsubsection{Theoretical performance of AMFMNs}

Since AMFMNs have symmetrical architectures in elevation and azimuth dimensions, we only analyze their filtering performance in the elevation dimension (Table 1). The SJNR of the AMFMN output-in $\tilde{r}_{\Sigma_{e}}$ in (17) can be derived from (8) as

$$
\begin{aligned}
\operatorname{SJNR}_{A_{e}} & =\frac{E\left[\mid\left(\boldsymbol{w}_{\Sigma}^{H}-w_{a} \boldsymbol{w}_{\Delta_{a}}^{H}\right) \boldsymbol{v}_{\left.\theta_{e_{T}},\left.\theta_{a_{T}} s_{T}\right|^{2}\right]}\right.}{E\left[\left|\left(\boldsymbol{w}_{\Sigma}^{H}-w_{a} \boldsymbol{w}_{\Delta_{a}}^{H}\right)\left(\boldsymbol{v}_{\theta_{e_{J}}, \theta_{a_{J}}} s_{J}+\boldsymbol{n}\right)\right|^{2}\right]} \\
& =\frac{P_{S}\left|\left(\boldsymbol{w}_{\Sigma}^{H}-w_{a} \boldsymbol{w}_{\Delta_{a}}^{H}\right) \boldsymbol{v}_{\theta_{e_{T}}, \theta_{a_{T}}}\right|^{2}}{\left(\boldsymbol{w}_{\Sigma}^{H}-w_{a} \boldsymbol{w}_{\Delta_{a}}^{H}\right) \boldsymbol{R}\left(\boldsymbol{w}_{\Sigma}-w_{a}^{*} \boldsymbol{w}_{\Delta_{a}}\right)} .
\end{aligned}
$$

By substituting (18) into (21), $S J N R_{A_{e}}$ can be approximately expressed as

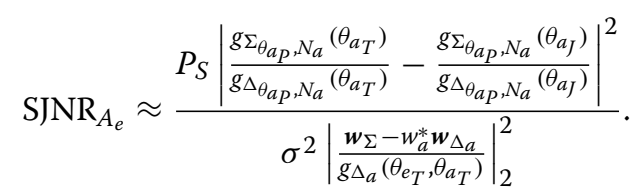


Table 1 Parameters for theoretical performance comparison

\begin{tabular}{lll}
\hline & Variable & Value \\
\hline Equivalent diameter of the target & $D$ & $10 \mathrm{~m}$ \\
Carrier wavelength & $\lambda_{c}$ & $0.03 \mathrm{~m}$ \\
Beam center & $\left(\theta_{e_{c^{\prime}}} \theta_{a_{c}}\right)$ & $\left(0^{\circ}, 0^{\circ}\right)$ \\
SNR of an antenna element & SNR & $0 \mathrm{~dB}$ \\
JNR of an antenna element & $\mathrm{JNR}$ & $20 \mathrm{~dB}$ \\
Column number of a single array & $N_{a}$ & 64 \\
Row number of a single array & $N_{e}$ & 64 \\
Array column number of DMA & $\frac{N_{a}}{2}$ & 32 \\
Array row number of DMA & $\frac{N_{e}}{2}$ & 32 \\
Array number of DMA & $N_{S}$ & 4 \\
Location of the target & $\left(x_{T}, y_{T}, z_{T}\right)$ & $(0,0,50000) \mathrm{m}$ \\
Location of SMN & $\left(x_{0}, y_{0}, z_{0}\right)$ & $(0,0,0) \mathrm{m}$ \\
Location of the first array of DMA & $\left(x_{1}, y_{1}, z_{1}\right)$ & $(-300,-300,0) \mathrm{m}$ \\
Location of the second array of DMA & $\left(x_{2}, y_{2}, z_{2}\right)$ & $(-300,300,0) \mathrm{m}$ \\
Location of the third array of DMA & $\left(x_{3}, y_{3}, z_{3}\right)$ & $(300,-300,0) \mathrm{m}$ \\
Location of the fourth array of DMA & $\left(x_{4}, y_{4}, z_{4}\right)$ & $(300,300,0) \mathrm{m}$ \\
\hline
\end{tabular}

\subsection{Maximum SJNR for array antennas}

External jamming including MLJ and SLJ on array antennas can be cancelled by an adaptive spatial filter under the MSJNR criterion. The coefficient of the MSJNR filter is derived in [9] as

$$
\boldsymbol{w}_{o}=\frac{\boldsymbol{R}^{-1} \boldsymbol{v}_{\theta_{e_{T}}, \theta_{a_{T}}}}{\boldsymbol{v}_{\theta_{e_{T}}, \theta_{a_{T}}} \boldsymbol{R}^{-1} \boldsymbol{v}_{\theta_{e_{T}}, \theta_{a_{T}}}} .
$$

The SJNR of the filtering output can be derived as

$$
\begin{aligned}
\operatorname{SJNR}_{M} & =\frac{\boldsymbol{w}_{o}^{H} \boldsymbol{v}_{\theta_{e_{T}}, \theta_{a_{T}}} \boldsymbol{v}_{\theta_{e_{T}}, \theta_{a_{T}}} \boldsymbol{w}_{o} P_{S}}{\boldsymbol{w}_{o}^{H} \boldsymbol{R} \boldsymbol{w}_{o}} \\
& =\frac{N_{s} N_{0} P_{S}}{\sigma^{2}}-\left|\rho_{S}\right|^{2}\left[\frac{N_{s}^{2} N_{0}^{2} P_{S} \sigma_{J}^{2}}{\left(\sigma^{2}+N_{S} N_{0} \sigma_{J}^{2}\right) \sigma^{2}}\right],
\end{aligned}
$$

where $\rho_{S}$ is the correlation coefficient of $\boldsymbol{v}_{\theta_{e_{T}}, \theta_{a_{T}}}$ and $\boldsymbol{v}_{\theta_{e},}, \theta_{a J}$, with the absolute value

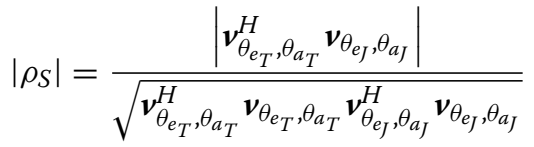

$$
\begin{aligned}
& =\left|\frac{\sin \frac{N_{S} N_{0} \pi\left(\sin \theta_{J}-\sin \theta_{T}\right)}{2}}{N_{S} N_{0} \sin \frac{\pi\left(\sin \theta_{J}-\sin \theta_{T}\right)}{2}}\right| \text {. }
\end{aligned}
$$

Equations (24) and (26) indicate that $\mathrm{SJNR}_{M}$ is a monotonically decreasing function of $\left|\rho_{S}\right|$, which is determined by the target direction, jammer direction, and antenna architecture.

\subsection{Applicability}

According to (22) and (24), the antenna gain of both filters achieves the minimum in the MLJ direction. Hence, MLJ can be cancelled, and the target echo in different directions can be reserved. By substituting $\theta_{e_{T}}=\theta_{e_{J}}, \theta_{a_{T}}=\theta_{a_{J}}$ into (21) and (24), the SJNR of the target echo arriving from the same direction as MLJ can be derived as

$$
\begin{aligned}
\operatorname{SJNR}_{A_{0}} & =\frac{\frac{P_{S}}{\sigma_{J}^{2}}}{1+\frac{\sigma^{2}}{\sigma_{J}^{2}} \frac{\left(\boldsymbol{w}_{\Sigma}^{H}-w_{a} \boldsymbol{w}_{\Delta a}^{H}\right)\left(\boldsymbol{w}_{\Sigma}-w_{a}^{*} \boldsymbol{w}_{\Delta_{a}}\right)}{\mid\left(\boldsymbol{w}_{\Sigma}^{H}-w_{a} \boldsymbol{w}_{\Delta_{a}}^{H}\right) \boldsymbol{v}_{\theta_{e_{T}},\left.\theta_{a_{T}}\right|^{2}}}}<\frac{P_{S}}{\sigma_{J}^{2}}, \\
\operatorname{SJNR}_{M_{0}} & =\frac{N_{S} N_{0} P_{S}}{\sigma^{2}+N_{S} N_{0} \sigma_{J}^{2}}<\frac{P_{S}}{\sigma_{J}^{2}},
\end{aligned}
$$

which means that the SJNRs are barely improved in this case.

Hence, the MLC techniques for single antennas can only deal with the condition that MLJ and the target echo arrive from different directions. To detect target echo arriving from the same direction as MLJ, new antenna architectures should be considered.

\section{Proposed method}

\subsection{Signal model}

The proposed radar system is illustrated in Fig. 2. There is a target accompanied by $I$ jammers in the mainlobe direction in the far end of this system.

An electromagnetic wave is transmitted from a preset array antenna of DMA and impinges on the aeroplane. The target echo and MLJs are received by all arrays of

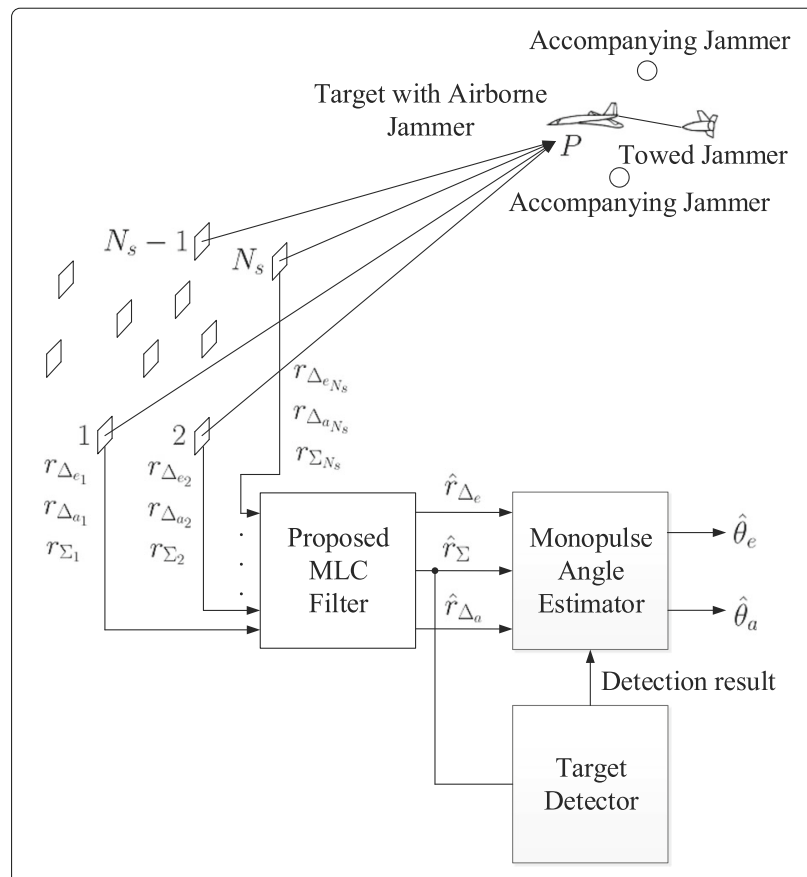

Fig. 2 Proposed target localization method for the DMA system 
the system. The time, frequency, and phase of the system are synchronized using the method proposed in $[17,18]$ before signal processing.

The common assumptions for establishing the signal model on DMA are as follows:

- $A_{1}$ : Antenna gain of each array. The planar arrays are placed parallel to each other. Their baselines are far less than the distance between the system and the target (or the ith jammer). Each array generates $\Sigma, \Delta_{e}$, and $\Delta_{a}$ beams simultaneously. The monopulse axes of these beams intersect at the predicted target position $P$. The distance between the predicted target and the actual target (or the ith jammer) is far less than the distance between the actual target (or the ith jammer) and the system. Thus, the $\Sigma$ (or $\Delta_{e}$, or $\Delta_{a}$ ) beam gain of each array on the actual target (or the ith jammer) can be approximated as identical, which is derived in Appendix A.

- $A_{2}$ : Power and correlation characteristics of the target echo.

Since the baselines of the system are far less than the distance between the target and the system, the power of the target echo arrived at each monopulse array is assumed to be equal. The correlation characteristic of the target echo on distributed antennas has been studied in [19]. This research indicates that the target echo received by each array of the proposed system can be assumed to be a partly correlated random variable. The correlation coefficient $c_{i, j}$ between the target echoes on the $i$ th and $j$ th distributed arrays can be modeled as

$$
c_{i, j}=J_{0}\left(\frac{2 \pi D \sin \frac{\Delta \beta_{i, j}}{2}}{\lambda_{c}}\right)+J_{2}\left(\frac{2 \pi D \sin \frac{\Delta \beta_{i, j}}{2}}{\lambda_{c}}\right) \leq 1,
$$

where $\lambda_{c}$ denotes the electromagnetic wavelength of the radar, $D$ denotes the diameter of the target, and $\Delta \beta_{i, j}$ denotes the angle between the $i$ th and $j$ th monopulse axes of DMA.

- $A_{3}$ : Model of MLJ and thermal noise MLJs from different jammers are modeled as independent random noise. Since the distributed antennas have short baselines, MLJ from the same jammer impinging on each distributed antenna can be modeled as a completely correlated signal [13]. The thermal noise of each $\Sigma$ (or $\Delta_{e}$, or $\Delta_{a}$ ) beam on DMA can be modeled as an i.i.d. Gaussian random variable with zero mean and variance $\sigma_{\Sigma}^{2}\left(\right.$ or $\left.\sigma_{\Delta_{e}}^{2}, \sigma_{\Delta_{a}}^{2}\right)$.

Suppose the position of the target is $T_{0}$ and that of the $i$ th jammer $(i>0)$ is $T_{i}$. By defining the distance between
$T_{i}$ and the $n_{s}$ th array as $r_{n_{s}, i}\left(n_{s}=1,2, \cdots, N_{s}\right)$, the steering vector of an ideal point target placed at $T_{i}$ on DMA can be expressed as

$$
\ell_{i}=\boldsymbol{d}_{i} \otimes v_{\theta_{e_{i}}, \theta_{a_{i}}},
$$

where $\boldsymbol{d}_{i}$ is defined as

$$
\boldsymbol{d}_{i}=\left[e^{-\mathrm{j} 2 \pi \frac{r_{1, i}}{\lambda_{c}}}, e^{-\mathrm{j} 2 \pi \frac{r_{2, i}}{\lambda_{c}}}, \ldots, e^{-\mathrm{j} 2 \pi \frac{r_{N_{s}, i}}{\lambda_{c}}}\right]^{T} .
$$

According to assumption $A_{1}$, the gain of the $\Sigma$ (or $\Delta_{e}$, or $\Delta_{a}$ ) beam on each array pointing at $T_{i}$ is considered to be identical. Hence, the beam gains at $T_{i}$ of each array are uniform and can be expressed as $g_{\Sigma}\left(\theta_{e_{i}}, \theta_{a_{i}}\right), g_{\Delta_{e}}\left(\theta_{e_{i}}, \theta_{a_{i}}\right)$, and $g_{\Delta_{a}}\left(\theta_{e_{i}}, \theta_{a_{i}}\right)$ respectively, where $\theta_{e_{i}}$ and $\theta_{a_{i}}$ are the elevation and azimuth angles of $T_{i}$ on a preset reference array.

According to assumption $A_{2}$, we model the correlation characteristic of the target echo with random vector $\eta$ that satisfies

$$
E\left[\boldsymbol{\eta} \eta^{H}\right]=C .
$$

The $(i, j)$-th element of the matrix $C$ is $c_{i j}$, which is modeled according to (29). Hence, the steering vector of the target echo on DMA is

$$
\tilde{\ell}_{0}=\left(\boldsymbol{\eta} \circ \boldsymbol{d}_{0}\right) \otimes \boldsymbol{v}_{\theta_{e_{0}}, \theta_{a_{0}}},
$$

whose corresponding $\Sigma, \Delta_{e}$, and $\Delta_{a}$ beam gain vectors on DMA can be obtained as

$$
\begin{aligned}
& \boldsymbol{g}_{\Sigma_{0}}=\left(\boldsymbol{\eta} \circ \boldsymbol{d}_{0}\right) \otimes\left(\boldsymbol{w}_{\Sigma}^{H} \boldsymbol{v}_{\theta_{e_{0}}, \theta_{a_{0}}}\right)=\left(\boldsymbol{\eta} \circ \boldsymbol{d}_{0}\right) g_{\Sigma}\left(\theta_{e_{0}}, \theta_{a_{0}}\right), \\
& \boldsymbol{g}_{\Delta_{e 0}}=\left(\boldsymbol{\eta} \circ \boldsymbol{d}_{0}\right) \otimes\left(\boldsymbol{w}_{\Delta_{e}}^{H} \boldsymbol{v}_{\theta_{e_{0}}, \theta_{a_{0}}}\right)=\left(\boldsymbol{\eta} \circ \boldsymbol{d}_{0}\right) g_{\Delta_{e}}\left(\theta_{e_{0}}, \theta_{a_{0}}\right), \\
& \boldsymbol{g}_{\Delta_{a 0}}=\left(\boldsymbol{\eta} \circ \boldsymbol{d}_{0}\right) \otimes\left(\boldsymbol{w}_{\Delta_{a}}^{H} \boldsymbol{v}_{\theta_{e_{0}}, \theta_{a_{0}}}\right)=\left(\boldsymbol{\eta} \circ \boldsymbol{d}_{0}\right) g_{\Delta_{a}}\left(\theta_{e_{0}}, \theta_{a_{0}}\right) .
\end{aligned}
$$

According to assumption $A_{3}$, the distributions of thermal noise corresponding to monopulse beams on DMA are

$$
\begin{aligned}
\boldsymbol{n}_{\Sigma} & \sim \mathcal{C N}\left(\mathbf{0}_{N_{s}}, \sigma_{\Sigma}^{2} \boldsymbol{I}_{N_{s} \times N_{s}}\right), \\
\boldsymbol{n}_{\Delta_{e}} & \sim \mathcal{C N}\left(\mathbf{0}_{N_{s}}, \sigma_{\Delta_{e}}^{2} \boldsymbol{I}_{N_{s} \times N_{s}}\right), \\
\boldsymbol{n}_{\Delta_{a}} & \sim \mathcal{C N}\left(\mathbf{0}_{N_{s}}, \sigma_{\Delta_{a}}^{2} \boldsymbol{I}_{N_{s} \times N_{s}}\right) .
\end{aligned}
$$

where $\boldsymbol{I}_{N_{s} \times N_{s}}$ is the identity matrix.

The steering vector of each MLJ is modeled according to (31). Hence, the signal vectors of monopulse beams on DMA are

$$
\begin{aligned}
\boldsymbol{r}_{\Sigma} & =\boldsymbol{g}_{\Sigma_{0}} s_{T}+\boldsymbol{G}_{\Sigma_{J}} \boldsymbol{s}_{J}+\boldsymbol{n}_{\Sigma}, \\
\boldsymbol{r}_{\Delta_{e}} & =\boldsymbol{g}_{\Delta_{e 0}} \boldsymbol{s}_{T}+\boldsymbol{G}_{\Delta_{e} J} \boldsymbol{s}_{J}+\boldsymbol{n}_{\Delta_{e}}, \\
\boldsymbol{r}_{\Delta_{a}} & =\boldsymbol{d}_{\Delta_{a 0}} s_{T}+\boldsymbol{G}_{\Delta_{a J}} \boldsymbol{s}_{J}+\boldsymbol{n}_{\Delta_{a}},
\end{aligned}
$$


where vector $\boldsymbol{s}_{J}=\left[s_{J_{1}}, s_{J_{2}}, \cdots, s_{I_{I}}\right]$ represents uncorrelated MLJ from the jammers. Moreover, matrices $\boldsymbol{G}_{\Sigma_{\jmath}}$, $\boldsymbol{G}_{\Delta_{e}}$, and $\boldsymbol{G}_{\Delta_{a J}}$ have the forms

$$
\begin{aligned}
\boldsymbol{G}_{\Sigma_{J}} & =\left[\boldsymbol{d}_{1} g_{\Sigma}\left(\theta_{e_{1}}, \theta_{a_{1}}\right), \cdots, \boldsymbol{d}_{I} g_{\Sigma}\left(\theta_{e_{I}}, \theta_{a_{I}}\right)\right], \\
\boldsymbol{G}_{\Delta_{e J}} & =\left[\boldsymbol{d}_{1} g_{\Delta_{e}}\left(\theta_{e_{1}}, \theta_{a_{1}}\right), \cdots, \boldsymbol{d}_{I} g_{\Delta_{e}}\left(\theta_{e_{I}}, \theta_{a_{I}}\right)\right], \\
\boldsymbol{G}_{\Delta_{a J}} & =\left[\boldsymbol{d}_{1} g_{\Delta_{a}}\left(\theta_{e_{1}}, \theta_{a_{1}}\right), \cdots, \boldsymbol{d}_{I} g_{\Delta_{a}}\left(\theta_{e_{I}}, \theta_{a_{I}}\right)\right] .
\end{aligned}
$$

\subsection{Proposed MLC filter}

According to (33), the steering vector of the target echo is an unknown random vector for a spatial filter. Hence, the classical MSJNR filter presented in [12] utilizes the standard steering vector model (31) and does not achieve optimum performance theoretically. The MMSE filter [12], the blind source separation (BSS) algorithm [20], or the eigen-projection processing and covariance matrix reconstruction algorithm (EPCMR)[10] can be employed to realize MLC. To detect and locate the target with higher performance, we propose a new MLC filter here to cancel MLJ.

Since the monopulse axes of all arrays are controlled to point at the expected target center, the gain of $\Sigma$ beams on the target echo is much higher than that of $\Delta_{e}$ (or $\Delta_{a}$ ) beams with a large probability. Hence, we select a signal from $\Sigma$ beams of DMA to design the spatial filter. By maximizing the power ratio of the received signal to jamming-plus-noise under the constraint of nulling MLJ, we propose the spatial filter $\boldsymbol{w}$ as

$$
\begin{array}{cl}
\min & \boldsymbol{w}^{H} \boldsymbol{R}_{\Sigma} \boldsymbol{w}, \\
\text { s.t. } & \boldsymbol{w}^{H} \boldsymbol{r}_{\Sigma}=1,
\end{array}
$$

where $\boldsymbol{R}_{\Sigma}$ is the covariance matrix of the received signal from $\Sigma$ beams on DMA in the form

$$
\boldsymbol{R}_{\Sigma} \triangleq E\left[\boldsymbol{r}_{\Sigma} \boldsymbol{r}_{\Sigma}^{H}\right]
$$

Since both the objective function (46) and constraint function (47) are convex, the optimization problem can be solved using the Lagrange multiplier method as

$$
\begin{aligned}
\xi & \triangleq \boldsymbol{w}^{H} \boldsymbol{R}_{\Sigma} \boldsymbol{w}+\lambda\left(\boldsymbol{w}^{H} \boldsymbol{r}_{\Sigma}-1\right), \\
\frac{\partial \xi}{\partial \boldsymbol{w}} & =\boldsymbol{R}_{\Sigma} \boldsymbol{w}+\lambda \boldsymbol{r}_{\Sigma}=0 .
\end{aligned}
$$

Combining (47) and (50), the solution of the optimization problem can be calculated as

$$
\boldsymbol{w}=\left(\boldsymbol{r}_{\Sigma}^{H} \boldsymbol{R}_{\Sigma}^{-1} \boldsymbol{r}_{\Sigma}\right)^{-1} \boldsymbol{R}_{\Sigma}^{-1} \boldsymbol{r}_{\Sigma}
$$

\subsection{Target detection and localization method}

\subsubsection{Detection method}

We detect the target from the filtering output of $\Sigma$ beams. The SJNR of the output at the time delay $\tau$ can be derived as

$$
\begin{aligned}
\operatorname{SJNR}(\tau) & =\frac{\boldsymbol{w}^{H}(\tau) \boldsymbol{r}_{\Sigma}(\tau) \boldsymbol{r}_{\Sigma}^{H}(\tau) \boldsymbol{w}(\tau)}{\boldsymbol{w}^{H}(\tau) \boldsymbol{R}_{\Sigma} \boldsymbol{w}(\tau)}=\frac{\left[\boldsymbol{r}_{\Sigma}^{H}(\tau) \boldsymbol{R}_{\Sigma}^{-1} \boldsymbol{r}_{\Sigma}(\tau)\right]^{2}}{\boldsymbol{r}_{\Sigma}^{H}(\tau) \boldsymbol{R}_{\Sigma}^{-1} \boldsymbol{R}_{\Sigma} \boldsymbol{R}_{\Sigma}^{-1} \boldsymbol{r}_{\Sigma}(\tau)} \\
& =\boldsymbol{r}_{\Sigma}^{H}(\tau) \boldsymbol{R}_{\Sigma}^{-1} \boldsymbol{r}_{\Sigma}(\tau) .
\end{aligned}
$$

The detection is performed using a constant false alarm rate (CFAR) criterion, whose detection threshold is determined by the preset false alarm rate. The background thermal noise is modeled to have zero mean, and a complex Gaussian random variable, whose power is a $\chi^{2}$-distribution random variable with two degrees of freedom.

Thus, the relationship of the threshold $\eta_{t h}$ and CFAR $P_{f}$ can be established as

$$
P\left(\operatorname{SJNR}(\tau)>\eta_{t h}\right)=1-\Psi\left(\eta_{t h}\right)=P_{f},
$$

where $\Psi$ is the cumulative distribution function (CDF) of $z$. The threshold $\eta_{t h}$ can be solved according to (53) as

$$
\eta_{t h}=\Psi_{2}^{-1}\left(1-P_{f}\right)
$$

where $\Psi_{2}^{-1}$ (.) is the inverse function of the CDF.

Then, the target detection can be performed considering the following rule:

$$
\left\{\begin{array}{l}
\operatorname{SJNR}(\tau)>\eta_{t h}, \text { accepted as target, } \\
\operatorname{SJNR}(\tau) \leq \eta_{t h}, \text { rejected as noise. }
\end{array}\right.
$$

\subsubsection{Localization method}

Theoretically, the target direction can be easily determined in the angle space using the method proposed in [21] when the target and jammers are sparsely distributed in the far field of each array. However, the signal sparsity will greatly decrease in the presence of MLJ. Hence, we propose a target direction estimation method according to a special antenna architecture of DMA. In particular, the same MLC filter (51) is simultaneously implemented for $\Sigma, \Delta_{e}$, and $\Delta_{a}$ beams of DMA as illustrated in Fig. 3. Thus, the output of the beams can be expressed as

$$
\hat{r}_{\Sigma}=\boldsymbol{w}^{H} \boldsymbol{r}_{\Sigma}, \hat{r}_{\Delta_{e}}=\boldsymbol{w}^{H} \boldsymbol{r}_{\Delta_{e}}, \hat{r}_{\Delta_{a}}=\boldsymbol{w}^{H} \boldsymbol{r}_{\Delta_{a}} .
$$

The voltage ratio of the filter output from $\Delta_{e}$ beams to that of $\Sigma$ beams can be derived as

$$
\hat{f}_{e}\left(\theta_{e_{0}}, \theta_{a_{0}}\right)=\frac{\boldsymbol{w}^{H} \boldsymbol{r}_{\Delta_{e}}}{\boldsymbol{w}^{H} \boldsymbol{r}_{\Sigma}}=\frac{\boldsymbol{w}^{H}\left(\boldsymbol{\eta} \circ \boldsymbol{d}_{0}\right) g_{\Delta_{e}}\left(\theta_{e_{0}}, \theta_{a_{0}}\right) s_{T}+\boldsymbol{w}^{H} \boldsymbol{n}_{\Delta_{e}}}{\boldsymbol{w}^{H}\left(\boldsymbol{\eta} \circ \boldsymbol{d}_{0}\right) g_{\Sigma}\left(\theta_{e_{0}}, \theta_{a_{0}}\right) s_{T}+\boldsymbol{w}^{H} \boldsymbol{n}_{\Sigma}} .
$$




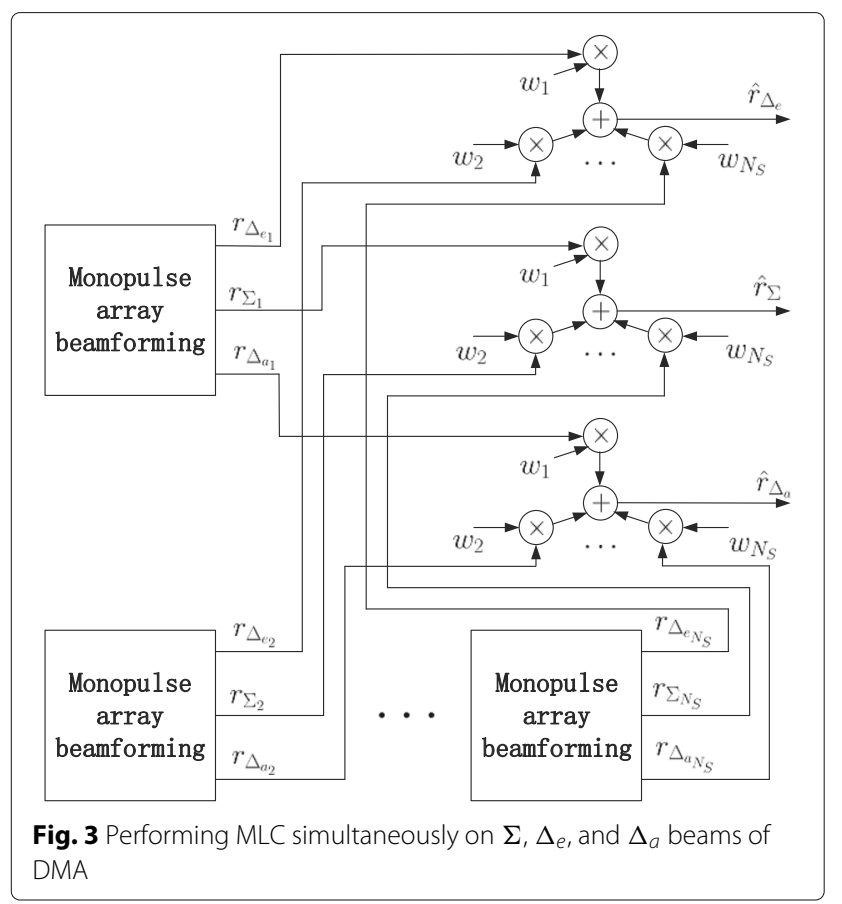

In practical application scenarios, the system is designed with a sufficient signal-to-noise ratio (SNR). Hence, the ratio $\hat{f}_{e}(t)$ can be approximately expressed as

$$
\hat{f}_{e}\left(\theta_{e_{0}}, \theta_{a_{0}}\right) \approx \frac{g_{\Delta_{e}}\left(\theta_{e_{0}}, \theta_{a_{0}}\right)}{g_{\Sigma}\left(\theta_{e_{0}}, \theta_{a_{0}}\right)} .
$$

Due to the symmetrical array architecture in the azimuth and elevation dimensions, the voltage ratio in the azimuth dimension can be expressed accordingly as

$$
\hat{f}_{a}\left(\theta_{e_{0}}, \theta_{a_{0}}\right)=\frac{\boldsymbol{w}_{p}^{H} \boldsymbol{r}_{\Delta_{a}}}{\boldsymbol{w}_{p}^{H} \boldsymbol{r}_{\Sigma}} \approx \frac{g_{\Delta_{a}}\left(\theta_{e_{0}}, \theta_{a_{0}}\right)}{g_{\Sigma}\left(\theta_{e_{0}}, \theta_{a_{0}}\right)} .
$$

Considering the monopulse principle $[1], \frac{g_{\Delta_{e}}\left(\theta_{e_{0}}, \theta_{a_{0}}\right)}{g_{\Sigma}\left(\theta_{e_{0}}, \theta_{a_{0}}\right)}$ in expression (58) is a bijective function of $\theta_{e_{0}}$ in the mainlobe direction. Hence, $\theta_{e_{0}}$ can be uniquely estimated using

$$
\hat{\theta}_{e_{0}}=\arg \min \left|\hat{f}_{e}\left(\theta_{e_{0}}, \theta_{a_{0}}\right)-\frac{g_{\Delta_{e}}\left(\theta_{e_{0}}, \theta_{a_{c}}\right)}{g_{\Sigma}\left(\theta_{e_{0}}, \theta_{a_{c}}\right)}\right| .
$$

Considering the same principle, $\theta_{a_{0}}$ can be uniquely estimated using

$$
\hat{\theta}_{a_{0}}=\arg \min \left|\hat{f}_{a}\left(\theta_{e_{0}}, \theta_{a_{0}}\right)-\frac{g_{\Delta_{a}}\left(\theta_{e_{c}}, \theta_{a_{0}}\right)}{g_{\Sigma}\left(\theta_{e_{c}}, \theta_{a_{0}}\right)}\right| .
$$

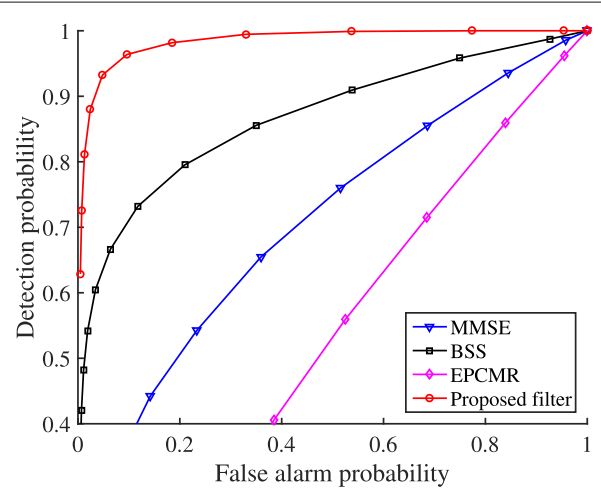

(a) $S N R=0 d B$

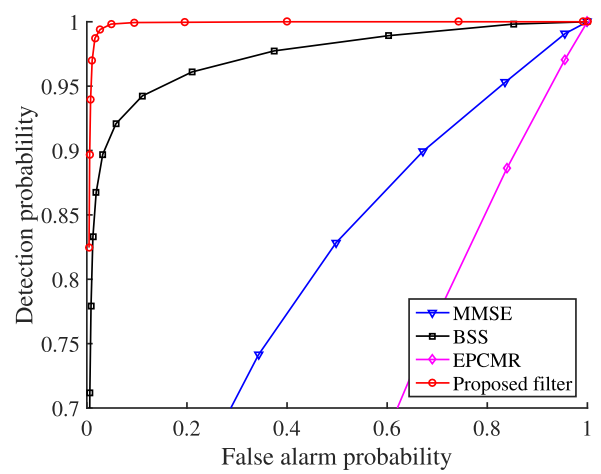

(c) $S N R=4 d B$

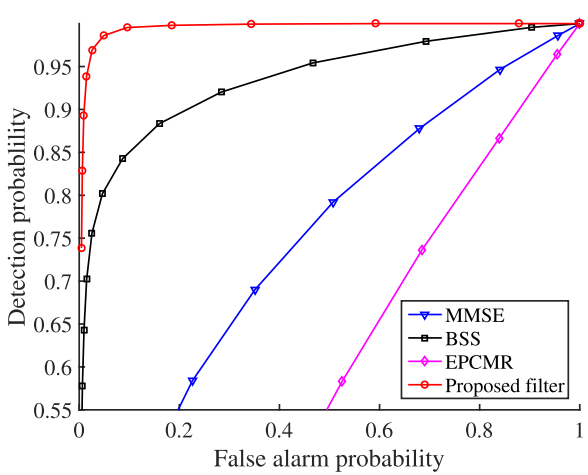

(b) $S N R=2 d B$

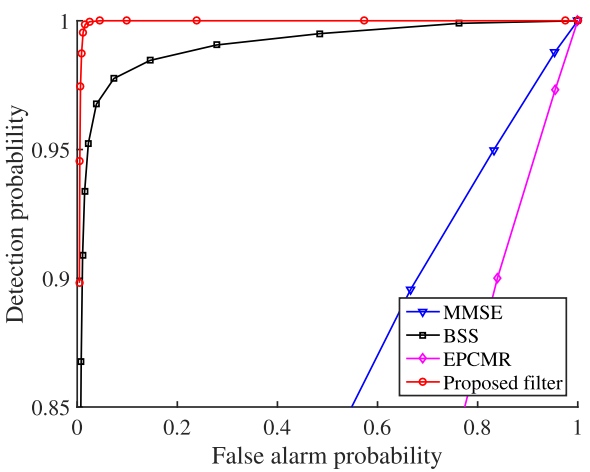

(d) $S N R=6 d B$

Fig. 4 ROC curves comparison under different SNRS 


\section{Results and discussion}

To illustrate the performance of the proposed target detection and localization method, we presents the numerical simulations on DMA system using the proposed filter and other signal separation methods including the MMSE filter[12] , BSS algorithm [20] and EPCMR method [10] for the same antenna architecture.

We consider a DMA system with $N_{s}=10$ identical planar arrays. The arrays are randomly placed on the $x y$ plane of the Cartesian coordinate system in the region $\{(x, y) \mid x \in[-300,300] \mathrm{m}, y \in[-300,300] \mathrm{m}\}$ according to a uniform distribution. The number of the elements in each column of the planar arrays is $N_{e}=32$, whereas that in each row is $N_{a}=64$. All the monopulse axes of DMA intersect at $P$, whose polar coordinate is $\theta_{e_{P}}=$ $\left.30^{\circ}, \theta_{a_{P}}=10^{\circ}, R_{P}=100000 \mathrm{~m}\right)$. Three mainlobe jammers are located at polar coordinates $\left(\theta_{e_{1}}=30.4^{\circ}, \theta_{a_{1}}=\right.$ $\left.10.2^{\circ}, R_{1}=100000 \mathrm{~m}\right),\left(\theta_{e_{2}}=29.9^{\circ}, \theta_{a_{2}}=9.9^{\circ}, R_{2}=\right.$ $100000 \mathrm{~m})$, and $\left(\theta_{e_{3}}=29.5^{\circ}, \theta_{a_{3}}=10.1^{\circ}, R_{3}=100000 \mathrm{~m}\right)$, respectively. The power ratio of MLJ to noise is $50 \mathrm{~dB}$ for each $\Sigma$ beam of the DMA system.

\subsection{Performance on target detection}

The target is placed at the position $\left(\theta_{e_{0}}=30^{\circ}, \theta_{a_{0}}=\right.$ $10^{\circ}, R_{0}=100000 \mathrm{~m}$ ) with the equivalent diameter $D=$
$10 \mathrm{~m}$. The SNRs of the target echo are set to different levels as $S N R=-3,0,3,6 \mathrm{~dB}$, respectively. For each SNR, 5000 independent Monte Carlo simulations are performed to evaluate the target detection performance using the proposed and other algorithms, including MMSE, BSS, and EPCMR. Figure 4 shows the receiver operating characteristic (ROC) curves of the detection probability $P_{d}$ versus the false alarm probability $P_{f}$ for each SNR on the same distributed monopulse arrays. It can be noticed from the figure that the proposed method has a higher detection performance compared with that of the other methods.

\subsection{Performance on target localization}

To demonstrate the target localization accuracy, the target is placed at different preset coordinates uniformly sampled from the $\operatorname{arc}\left(\theta_{e_{0}} \in\left[29^{\circ}, 31^{\circ}\right], \theta_{a_{0}}=10^{\circ}, R_{0}=\right.$ $100000 \mathrm{~m})$ and $\left(\theta_{e_{0}}=30^{\circ}, \theta_{a_{0}} \in\left[9.5^{\circ}, 10.5^{\circ}\right], R_{0}=\right.$ $100000 \mathrm{~m}$ ) with the sampling spacing $\theta_{s}=0.05^{\circ}$. The SNR of the target echo is set to SNR $=6 \mathrm{~dB}$. At each sampled position, MLJ is cancelled using the proposed filter and other methods including MMSE, BSS, and EPCMR. Relative angle estimation is performed on the filtering output according to monopulse estimation principle. For each filtering algorithm, 5000 independent Monte Carlo

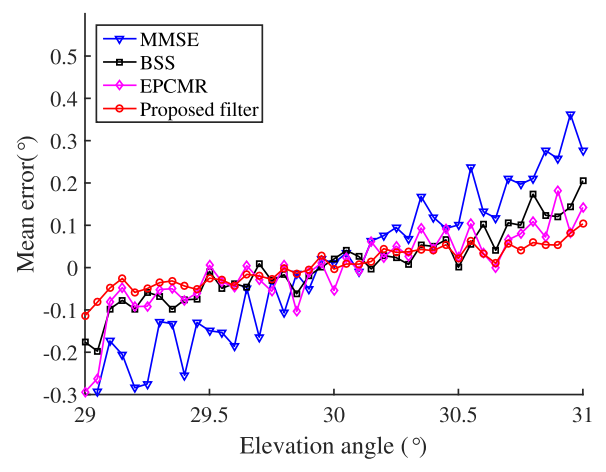

(a)

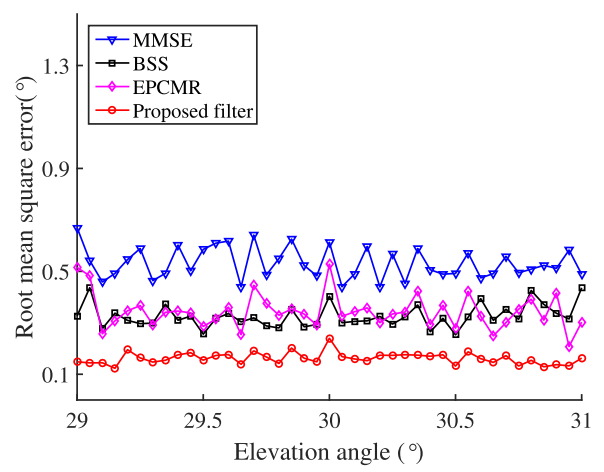

(c)

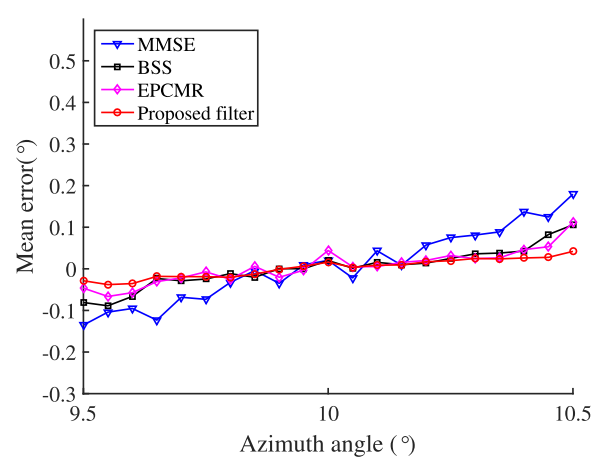

(b)

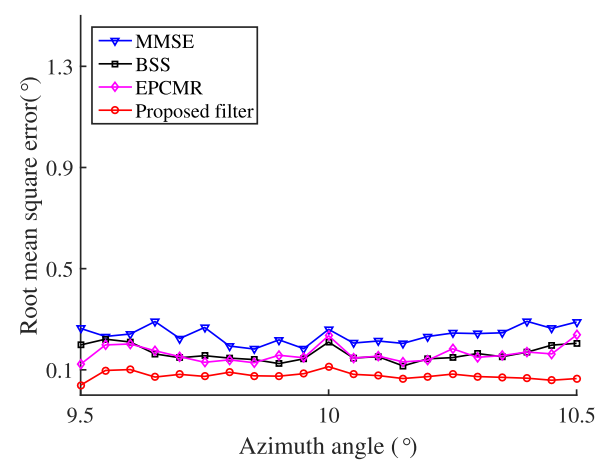

(d)

Fig. 5 Relative angle estimation performances comparison 
simulations are taken. The mean error and the root mean square error of the estimated relative angle under each filtering method are shown in Fig. 5, which indicate that the monopulse estimator under the proposed filter can get higher estimation accuracy than that under other filtering method.

\section{Conclusion}

In this paper, a MLC method on DMA was proposed, which is a new antenna architecture to deal with this problem. In this antenna architecture, the target echo has different steering vectors with MLJ even when they arrive from the same direction, which prevents the target echo from being cancelled by the MLC filter. Moreover, the relative angle of the target can be accurately measured since the monopulse ratio is maintained when using the proposed filtering method. According to the performed Monte Carlo simulations, the system can detect and locate the target with a higher accuracy when using the proposed filter compared with that when using other filtering algorithms. Moreover, the simple antenna architecture makes the proposed method applicable to practical tracking radar systems.

\section{Abbreviations \\ AMFMNs: Adaptive MLC filters for monopulse networks; BSS: Blind source separation; CDF: Cumulative distribution function; CFAR: Constant false alarm rate; DMA: Distributed monopulse antennas; EPCMR: Eigen-projection processing and covariance matrix reconstruction; MLC: Mainlobe cancellation; MLJ: Mainlobe jamming; MMSE: Minimizing the mean square error; MSJNR: Maximizing SJNR; RSV: Relative steering vector; SJNR: Signal to jamming-plus-noise ratio; SLC: Sidelobe cancellation; SLJ: Sidelobe jamming SNR: Signal-to-noise ratio; ULA: Uniform linear array}

\section{Acknowledgements}

The authors thank for the valuable and constructive comments from the editor and reviewers.

\section{Authors' contributions}

QS conceived of the algorithm and designed the experiments. QZ and XH performed the experiments, analyzed the results, and drafted the manuscript. $\mathrm{QG}$ and $\mathrm{XH}$ revised the manuscript. All authors read and approved the final manuscript.

\section{Funding}

This research received no specific grant from any funding agency in the public, commercial, or not-for-profit sectors.

\section{Availability of data and materials}

The data and materials are available for reviewers and readers.

\section{Competing interests}

The authors declare that they have no competing interests.

\author{
Author details \\ ${ }^{1}$ Air Force Engineering University, 710051 Xi'an, China. ${ }^{2}$ Air Force
}

Communications NCO Academy, 116600 Dalian, China.

Received: 27 September 2019 Revised: 30 November 2019 Accepted: 8 January 2020

Published online: 16 January 2020

\section{References}

1. S. M. Sherman, Monopulse Principles and Techniques. (MA : Artech House, Dedham, 1984)

2. N. Li, Y. Zhang, A survey of radar ecm and eccm. IEEE Trans. Aerosp. Electron. Syst. 31(3), 1110-1120 (1995). https://doi.org/10.1109/7.395232

3. A. Abouelfadl, A. M. Samir, F. M. Ahmed, A. H. Asseesy, in 201633rd National Radio Science Conference (NRSC). Performance analysis of Ifm pulse compression radar under effect of convolution noise jamming (IEEE, 2016). https://doi.org/10.1109/nrsc.2016.7450840

4. P. Howells, Explorations in fixed and adaptive resolution at ge and surc IEEE Trans. Antennas Propag. 24(5), 575-584 (1976). https://doi.org/10. 1109/TAP.1976.1141401

5. R. L. Haupt, Antenna Arrays: A Computational Approach. (USA:Wiley, New York, 2010)

6. K. Yu, D. J. Murrow, Adaptive digital beamforming for angle estimation in jamming. IEEE Trans. Aerosp. Electron. Syst. 37(2), 508-523 (2001). https:// doi.org/10.1109/7.937465

7. S. Moon, D. Han, H. Oh, M. Cho, in IEEE Military Communications Conference, 2003. MILCOM 2003. Monopulse angle estimation with constrained adaptive beamforming using simple mainlobe maintenance technique, vol. 2 (IEEE, 2003), pp. 1365-13692. https://doi.org/10.1109/ milcom.2003.1290425

8. R. Li, C. Rao, L. Dai, Y. Wang, Combining sum-difference and auxiliary beams for adaptive monopulse in jamming. J. Syst. Eng. Electron. 24(3), 372-381 (2013). https://doi.org/10.1109/JSEE.2013.00046

9. H. L. Van Trees, Optimum Array Processing-Part IV of Detection, Estimation, and Modulation Theory. (USA:Wiley, New York, 2002)

10. X. Yang, Z. Zhang, T. Zeng, T. Long, T. K. Sarkar, Mainlobe interference suppression based on eigen-projection processing and covariance matrix reconstruction. IEEE Antennas Wirel. Propag. Lett. 13, 1369-1372 (2014) https://doi.org/10.1109/LAWP.2014.2339224

11. R. L. Fante, R. M. Davis, T. P. Guella, Wideband cancellation of multiple mainbeam jammers. IEEE Antennas Wirel. Propag. Lett. 44(10), 1402-1413 (1996). https://doi.org/10.1109/8.537336

12. X. Yang, P. Yin, T. Zeng, T. K. Sarkar, Applying auxiliary array to suppress mainlobe interference for ground-based radar. IEEE Antennas Wirel. Propag. Lett. 12, 433-436 (2013). https://doi.org/10.1109/LAWP.2013. 2254698

13. S. Zhao, L. Zhang, Y. Zhou, N. Liu, Signal fusion-based algorithms to discriminate between radar targets and deception jamming in distributed multiple-radar architectures. IEEE Sensors J. 15(11),6697-6706 (2015). https://doi.org/10.1109/JSEN.2015.2440769

14. Q. Zhang, F. Gao, Q. Sun, X. Wang, Mainlobe jamming cancelation method for distributed monopulse arrays. Sci. China Inf. Sci. 61 (2018). https://doi.org/10.1007/s11432-017-9274-3

15. T. T. Taylor, Design of line-source antennas for narrow beamwidth and low side lobes. Trans. IRE Prof. Group Antennas Propag. 3(1), 16-28 (1955) https://doi.org/10.1109/TPGAP.1955.5720407

16. E. T. Bayliss, Design of monopulse antenna difference patterns with low sidelobes. Bell Syst. Tech. J. 47(5), 623-650 (1968). https://doi.org/10. 1002/j.1538-7305.1968.tb00056.x

17. W. Wang, Gps-based time phase synchronization processing for distributed sar. IEEE Trans. Aerosp. Electron. Syst. 45(3), 1040-1051 (2009). https://doi.org/10.1109/TAES.2009.5259181

18. Y. Yang, R. S. Blum, Phase synchronization for coherent mimo radar: Algorithms and their analysis. IEEE Trans. Sig. Process. 59(11), 5538-5557 (2011). https://doi.org/10.1109/TSP.2011.2162509

19. S. Zhou, H. Liu, Y. Zhao, L. Hu, Target spatial and frequency scattering diversity property for diversity mimo radar. Sig. Process. 91, 269-276 (2011). https://doi.org/10.1016/j.sigpro.2010.07.004

20. W. Liu, D. P. Mandic, A normalised kurtosis based algorithm for blind source extraction from noisy measurements. Sig. Process. 5, 1580-1585 (2005). https://doi.org/10.1016/j.sigpro.2005.09.001

21. H. Xie, F. Gao, S. Zhang, S. Jin, A unified transmission strategy for tdd/fdd massive mimo systems with spatial basis expansion model. IEEE Trans. Veh. Technol. 66(4), 3170-3184 (2017). https://doi.org/10.1109/TVT.2016. 2594706

\section{Publisher's Note}

Springer Nature remains neutral with regard to jurisdictional claims in published maps and institutional affiliations. 